\title{
AUTOMATIC ASSEMBLY LINE SYSTEM OF GARMENT BRACKET BASED ON PLC
}

\author{
Xinyun ZHOU \\ Gannan Normal University, Gannan, China \\ Email: zttx72@163.com
}

\begin{abstract}
The purpose is to study the automatic assembly line system of garment bracket based on PLC. Assembly is an indispensable part of the manufacturing process. The improvement of assembly efficiency is one of the key problems in manufacturing industry. In order to realize the automatic assembly of PLC clothing board, a kind of assembly system based on PLC is proposed. It is analyzed from two aspects of hardware and software. The results show that the system has the advantages of high precision, high efficiency, easy operation and high reliability. Therefore, it is concluded that the system can meet the requirements of the automatic assembly line system of the clothing. It is a practical system with good maneuverability.
\end{abstract}

Keywords: Yarn Board, PLC, Automatic Assembly.

\section{Introduction}

The most important feature of the modern automatic assembly is the typical mechanism, and the components can be better used in general (that is, flexible assembly). Different assembly objects use the same automatic assembly machine, and only part of the fixture is different. The degree of generalization of components is even higher than some combination machines [1]. Based on PLC, an automatic assembly line for the yarn board is designed.

\section{State of the art}

At the beginning of the 20th century, Ford Motor Company established the first assembly line [2]. Transport belt mobile car assembly line carefully divided process, and realized professional assembly.

The assembly time is decreased by $90 \%$. In recent years, some progress has been made in automated assembly technology in our country. Some semiautomatic and automatic assembly lines have been designed and imported. The professional scientific research and design team engaged in automatic assembly technology gradually formed. A specialized academic institution has been set up. As an emerging profession, automated assembly technology is taking shape. Compared with manual assembly, automated assembly has the following advantages [3]. It has high efficiency and low cost. The mechanical device was used to do the tedious work, which greatly reduced the labor intensity of the workers. It will not cause product quality defects, due to fatigue, negligence, technical unskilled and other factors. The assembly area occupied by automated assembly equipment is much smaller than the production area occupied by manual assembly under the same conditions. In electronics, chemistry, aerospace, defense and other industries, many assembly operations need to be in a special environment. Human beings are difficult to enter it. Only automated assembly can ensure production safety [4]. The assembly line that designed in this paper can guarantee the assembly quality, improve the efficiency of labor production, reduce the production cost and improve the working conditions.

\section{Methodology}

\subsection{The process flow and the control requirements}

After the automatic assembly line of the yarn board is made, the automatic feeding, automatic gluing, automatic bonding and automatic blanking of the yarn releasing plate can be realized. In addition to the four assembly mechanisms, the automatic assembly line has six position turning tables, pneumatic systems, hydraulic systems, sensors, PLC control cabinets, and servo control systems. These devices are classified. It shows that the assembly line is composed of three parts: mechanical system, transmission system, control system and auxiliary system. They permeate each other and complement each other.

After the preliminary design, the automatic assembly line of the yarn stop needs 7 hydraulic cylinders, 13 cylinders and 20 electromagnetic valves. They need to be optimized to achieve 
coordinated design. The hydraulic system is composed of an oil pump, an electric motor, hydraulic cylinders, a traveling switch, 7 two position four - way electromagnetic valves and an indicator lamp. The pneumatic system consists of 13 cylinders, stroke switches, 13 electromagnetic air valves and indicator lights. When the button switch or trip switch sends the signal back to the PLC input terminal, and after the PLC processing, the action control signal is sent to drive the on-off of the solenoid valve coil. The movement direction of hydraulic oil causes the hydraulic cylinder piston to act accordingly.

The mechanical system is the main part of the yarn blocking plate automatic assembly line part, including the glue machine 1 , glue machine 2 , porcelain bonding machine 1 , porcelain bonding machine 2, positioning mechanism and clamp mechanism. And they are fixed on and around a 6position rotary table. The gluing mechanism and the porcelain adhering mechanism depend on the left and right movement of the hydraulic cylinder and the piston of the air pressure cylinder to make the assembly mechanism move, such as the lifting of the porcelain head and the advancing and retreating of the gluing motor.
The positioning mechanism is a positioning pin controlled by an air pressure cylinder. And when each turning station is moved to a work station, the positioning pin moves forward to the hole on the accompanying clamp and blocks the turning table, so as to prevent the turning of the turning table in the assembly process. The clamping mechanism is six accompanying elastic clamps which are uniformly fixed on the rotary table, they are released in the process of feeding and blanking, and the gluing and sticking process is the clamping state.

\subsection{The hardware design of PLC}

The hardware design of PLC system means that the controller, actuator, sensor, power unit and mechanical device are selected or manufactured to meet the requirement of process. In addition to the high performance of individual components, it emphasizes the coordination and coordination between the five parts of the controller, actuators, sensors, power units, and mechanical devices. The structure of the PLC hardware system is shown in Figure 1.

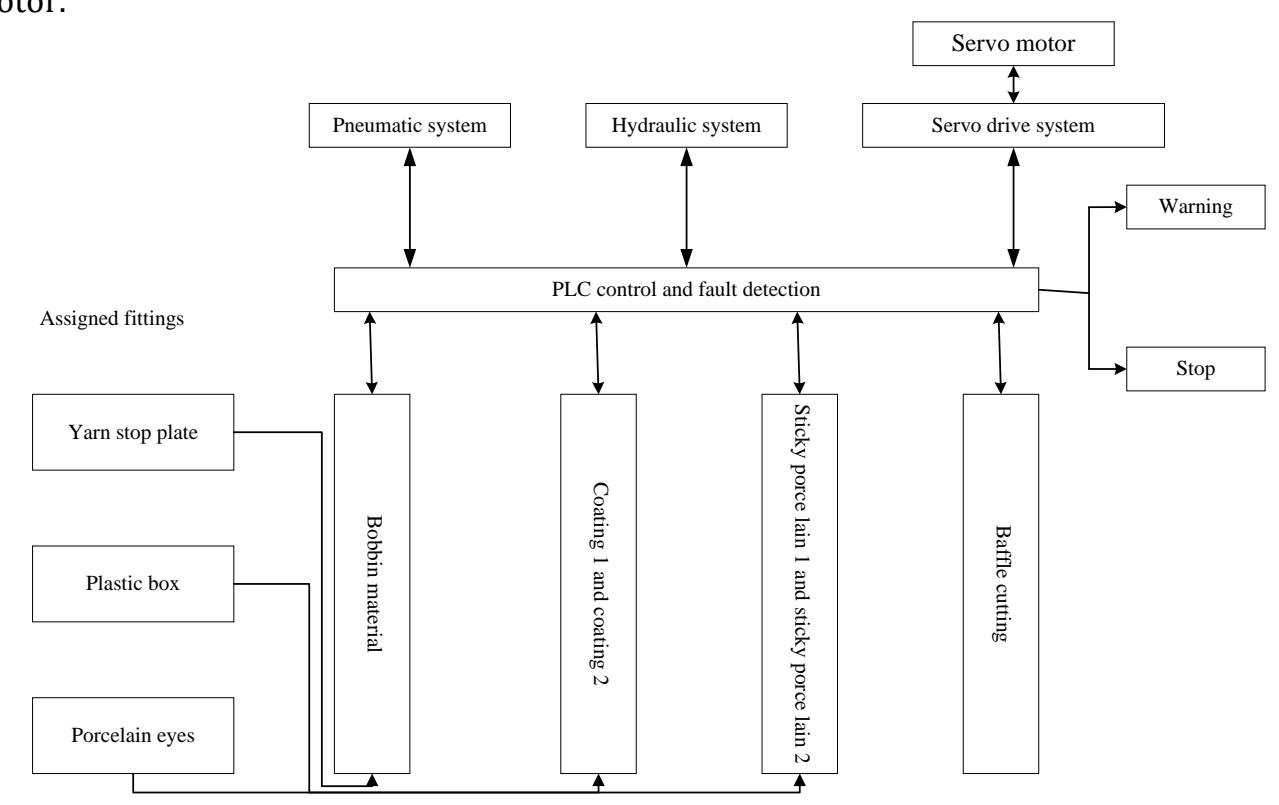

Fig. 1 The structure of PLC hardware system

According to the composition of the automatic assembly line structure and process requirements, the design is divided into the following aspects. The automatic assembly line control system is based on the programmable controller (PLC), receives the control commands from the host computer, and sends signals to the execution devices. The feedback signal from the whole system is mainly the signal sent by the system body to the limit position, that is, the signal sent by the limit position switch in the forward and reverse direction.

The operation panel includes all the control requirements for the automatic assembly line of the baffle plate. They are power switch, emergency stop button, working mode switching, glue, clay, feeding, cutting, cylinder front and rear, upper and lower movement buttons, lights and so on. Servo motor, oil pump motor and glue coating motor are important parts of the automatic assembly line. They respectively control the movement of the turret, the relative feeding motion of the hydraulic cylinder and the pneumatic cylinder, and the rotation movement of the gluing head. For example, the PLC sends a signal to the servo motor that controls the turret, 
and receives feedback from the motor via a servo drive system.

The pressure control loop operation of pneumatic system is shown as follows: the gas source generates the compressed air. After triad processing, by twoposition four-way valve and a one-way throttle valve, it enters the feeding cylinder, the positioning cylinder, the coated rotary cylinder 1 , the coated rotary cylinder 2 , the coated mobile cylinder 1 , the coated mobile cylinder 2 , the adhesive porcelain cylinder 1 , the porcelain cylinder 1 , the porcelain cylinder 2, blanking head cylinder, blanking moving cylinder, and blanking stirring cylinder.

In industrial control, the commonly used control systems are numerical control (NC) systems, industrial computers, single-chip microcomputer and PLC. The NC system, with the computer as the core, has a fairly comprehensive function. It can carry out a lot of precision machining operations, and the control program is relatively complex. It is suitable for the production and manufacturing process with quite high requirements on the manufacture precision and that cannot be completed artificially, whose price cost is also high. Industrial personal computer (IPC), as the name suggests, is a control system used in industrial environments. It also takes the computer as the core, with more powerful functions and friendly man-machine interface, which can make use of the advanced programming language. However, its antiinterference ability needs to be strengthened, and in some extremely harsh production environments, its stability and reliability is not satisfactory. As a result, it can only be used in a good production environment.

The single-chip microcomputer (SCM), compared with the first two kinds, has much smaller volume. Its power consumption is naturally much less, and the area occupied is much less.

Similarly, due to the size restrictions, its function is comparatively the weakest. In consequence, it can only use the assembly language for programming, the readability is poor, and the development cycle is long. PLC has the characteristics of easy to learn, strong function, high cost performance, and complete hardware. It is convenient for users to use, which has the advantages of strong adaptability, high reliability, strong anti-interference ability, short design cycle of the system, less installation and debugging work, small volume, low energy consumption and so on. Compared with various systems realizing the sequence control which is currently used in the industrial process, it has obvious advantages. Therefore, with comprehensive consideration, the PLC is selected as the control system of the assembly line of the garment bracket.

\subsection{The software design of PLC}

PLC is a control device that performs logic functions. CPU is used to complete the logic operation, and the memory is used to save these completed operations [5]. PLC equivalent circuit can be divided into the following three parts: input part, the internal control circuit and the output part. The structure of the PLC is shown in Figure 2.

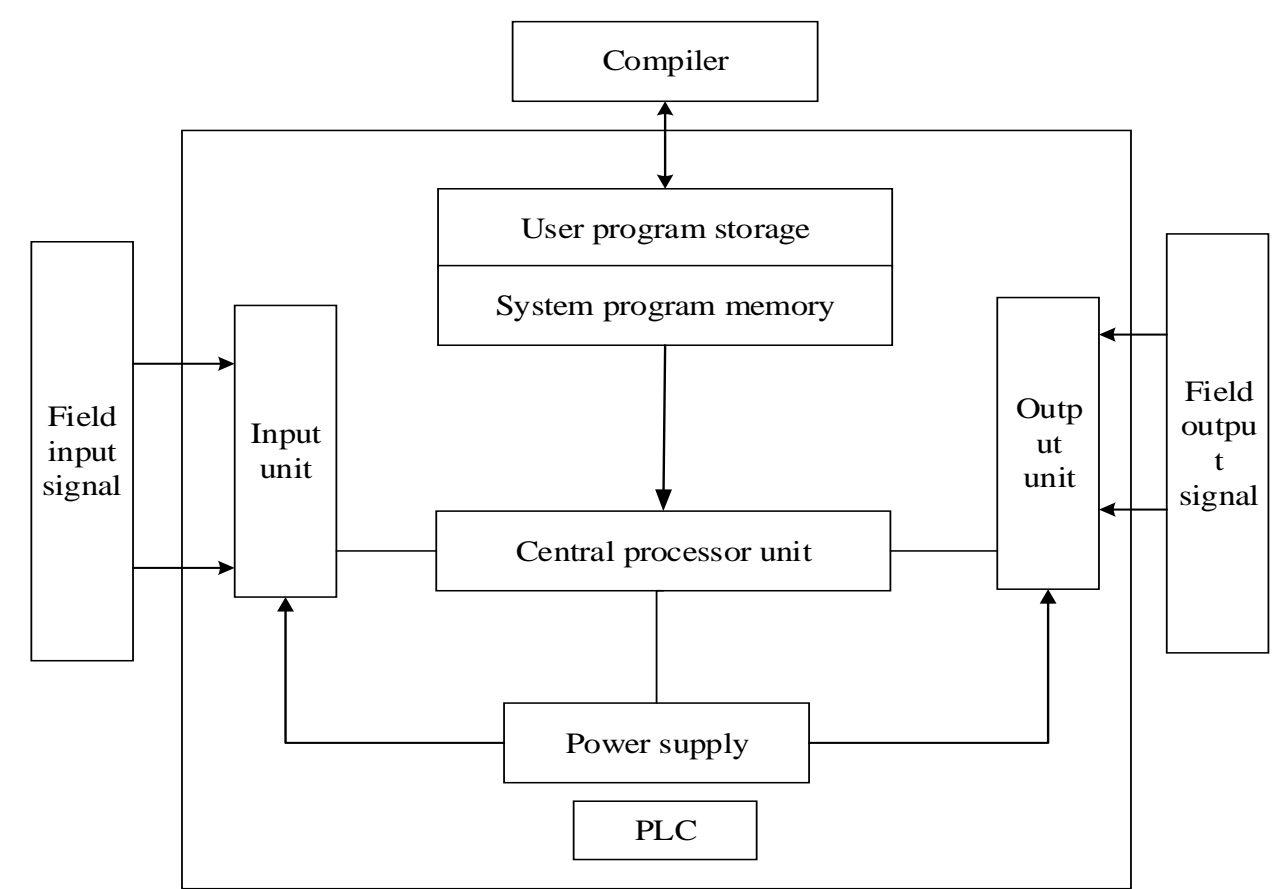

Fig. 2. The structure of the PLC 
PLC takes the cycle of scanning work. The cycle scan can be roughly divided into four sessions: self-diagnosis, the implementation of user programs, I / 0 refresh, peripheral port services. Before each scan begins, the CPU performs a reset watchdog timer, a hardware check, and a user memory check.

If there is an abnormality, the PLC will light up the fault indicator. In addition, it can judge and show the nature of the fault. The time of selfdiagnosis is generally fixed, and there are differences between different PLC. In a certain order, CPU scans and runs user programs.

The selection of PLC is mainly to meet the functional requirements, and it is more reliable, convenient maintenance and cost-effective. In addition to the basic functions of the general PLC, the selected PLC should meet the following requirements. First of all, the turntable is to rely on servo drive to control the walk and positioning. Therefore, PLC must have the pulse output function. Second, as the core components of motion control, PLC can communicate with the host computer. Therefore, PLC must have the ability to communicate with the computer function. At present, the hardware structure of PLC is mainly divided into two kinds: integral type and modular type. In a better working environment, such as a more fixed environment, priority should be given to the use of integrated PLC. In contrast, modules should be considered priority when the working environment is poor. The working environment of the automatic assembly line of the yarn stop is good, so the integral PLC is selected. PLC has three I/0 types: switching, analog, and special functions. The main switches are stroke switch, button, start stop and indicator light. Analog signals refer to continuous signals such as temperature and current. Special signals refer to signals such as positioning and fast input. In the automatic assembly line of the yarn stop, the type of I/0 is mainly the switching value. I/0 points: PLC input and output points in the selection of PLC is a very important indicator. The first principle of selection is to determine the number of $\mathrm{I} / 0$ points required to complete the project. Then, under the premise of satisfying the conditions, the PLC with low price and good reliability is selected.

According to the point number classification, PLC input and output points can be divided into three categories. The points below 128 are small PLC. The points between 128-512 are mediumsized PLC. The points above 512 are large PLC.

After calculation, it is concluded that the automatic assembly line of the bezel is about 57 , the output point is 41 , and the total $\mathrm{I} / 0$ points is 98. Taking into account the $20 \%$ of the points margin, the total number of points less than 128 were selected. Therefore, the small PLC is selected. In accordance with the region, PLC manufacturers have three major factions, that is, the United States PLC, the European PLC, and Japan's PLC. PLC manufacturers in the United States and Europe are mainly good at manufacturing large and medium-sized PLC. However, in the small PLC research and production, Japan has a good reputation. In summary, Omron's small, high-performance PLCCP1H-X40DT-D is selected. It has 24 input points and 16 output points. It can select the relay output or transistor output. There are 7 extension units.

The maximum switching amount is 320 points, and the maximum analog quantity is 37 . The PLC communicates with the host computer by using the USB port. Ladder diagram with the structure of the function block Text language programming is used. There are two serial communication ports (RS-232C or RS-422A / 485 option board to choose from), which can be easily achieved with the man-machine interface between the connection.

\subsection{PLC program flow}

The automatic assembly line PLC program is written in CX-Program. The CX-Program is a ladder programming software developed by Omron for C Series PLCs. In the Windows system, the ladder diagram of the programming, monitoring and control functions can be achieved, especially good at the preparation of large programs [6]. Here, the ladder method and the sequential control design method are mainly used to write the program. In CX-Programme, a new project is created first, and then the PLC program is started. The PLC program includes manual programs and automatic programs. The manual procedures are mainly used in the installation, debugging and maintenance of equipment, and each part of the function needs to press the corresponding button manually. An automatic program is a program when it is working. It realizes the continuous movement of the whole assembly process by controlling the main program and calling subprogram of each assembly station. The subroutine mainly has feeding subroutine, GI, glue subroutine, G2 and G3, stick porcelain subroutine G4 and G5, blanking subroutine G6.

\section{Result analysis and discussion}

\subsection{Laboratory debugging and site commissioning}

The simulation debugging is to use the auxiliary relay instead of the input relay (that is, imitate the external input relay action) to debug the program.

The simulation and debugging of the on-off control system is very convenient, which can be used to simulate the actual input signal by switching on and off. By observing the output of the LEDs showing the status of 
the relay, the simulation of the ladder diagram's implementation is understood. By taking advantage of the power of the PLC, the switch contacts in the program are used to make the program run.

After the program simulation, the hardware and software are tested on the equipment to observe the assembly line. The manual mode should be used preferentially when debugging. If each module is functioning properly, the automatic mode operation is adopted.

\subsection{Result}

The work environment of the automatic assembly line is very good. The automatic assembly line of the baffle plate is about to enter 57 points, the output point is 41 , and the total I / 0 points is 98 . Taking into account the $20 \%$ of the remaining points, and the total number of points must be less than 128, Omron's small high-performance
PLCCP1H-X40DT-D was the final selection. A set of automatic feeding, automatic gluing, automatic bonding and automatic blanking assembly system based on PLC has been designed.

The assembly function of the yarn stop plate is introduced, and the assembly system of the automatic assembly line is designed on the whole, as shown in Figure 3. The process flow is shown in Figure 4.

The assembly line is an automatic assembly line with PLC as the core and the auxiliary blanking machine as a whole. Then, after the design of the structure and function, the software of the assembly line is designed. It includes the selection of PLC, the design and distribution of I/O points, the programming and debugging of the program, and so on. Among them, the PLC program is modular, so that the program structure is more clear and easy to understand, which is convenient for future inspection and modification. On the whole, the automatic assembly line of the yarn stop plate has the advantages of high assembly accuracy, high efficiency, convenient operation and high reliability.

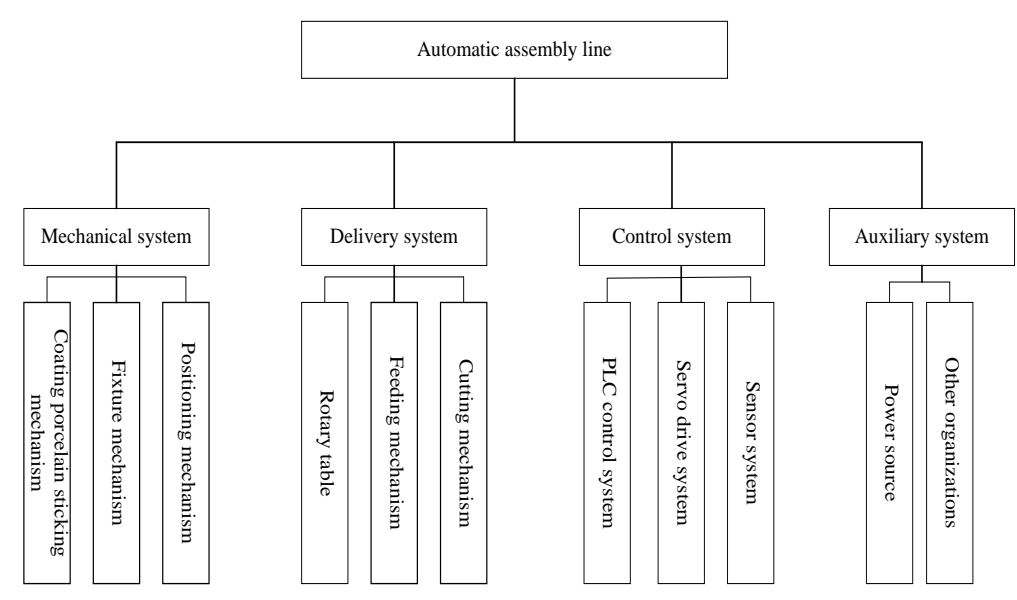

Fig. 3 The structure of the automatic assembly line of the baffle plate

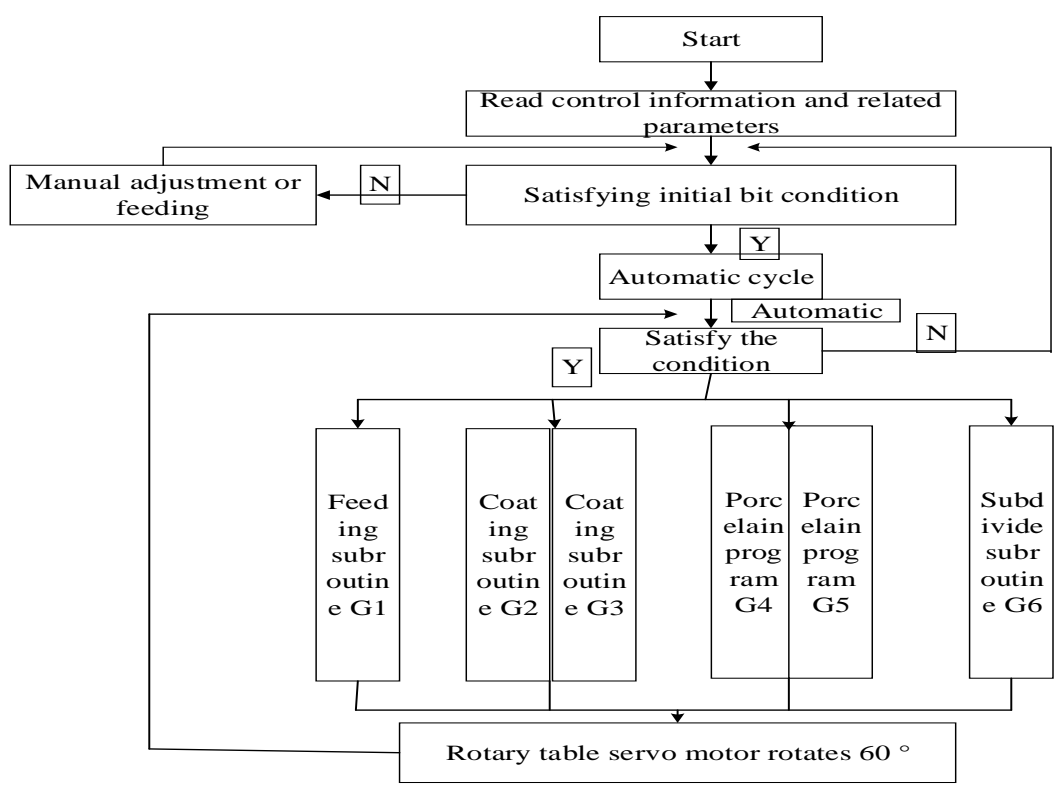

Fig. 4 PLC general program flow chart 


\section{Conclusion}

Automated assembly is a very complicated technology, which occupies a very important position in the machine building industry. It is not only an important turning point in the development of automated production, but also a means of transition to unmanned production. In the future, there may be some revolutionary changes in assembly methods, such as flexible assembly and inspection assembly. In assembly, it can detect the qualification of the product according to the input of product information. There are even more intelligent devices. It can not only detect defects in products, but also put forward some suggestions for improvement, and automatically re processing.

Finally, the finished product is assembled. The future process of production may be fully automated and intelligent. The equipment has been able to accomplish many things by itself. Automatic assembly line has been applied in many aspects of modern assembly technology, such as PLC control system, servo drive positioning system and so on. All technologies cooperate with each other and support each other.

\section{References}

[1]Michalos, George, et al. "Decision making logic for flexible assembly lines reconfiguration." Robotics and Computer-Integrated Manufacturing 37 (2016): 233-250. https://doi.org/10.1016/j.rcim.2015.04.006 [2]in, Byoungho. "Apparel industry in East Asian newly industrialized countries: Competitive advantage, challenge and implications." Journal of Fashion Marketing and Management: An International Journal 8.2 (2004): 230-244. https://doi.org/10.1108/13612020410537915

[3]Matt, Dominik T., and Erwin Rauch. "Designing assembly lines for mass customization production systems." Mass Customized Manufacturing: Theoretical Concepts and Practical Approaches (2017). https://doi.org/10.1201/9781315398983-3

[4]Marsillac, Erika, and James Jungbae Roh. "Connecting product design, process and supply chain decisions to strengthen global supply chain capabilities." International Journal of Production Economics 147 (2014): 317-329. https://doi.org/10.1016/j.ijpe.2013.04.011

[5]Cachapa, Daniel, et al. "An approach for integrating real and virtual production automation devices applying the serviceoriented architecture paradigm." Emerging Technologies and Factory Automation, 2007. ETFA. IEEE Conference on. IEEE, 2007.https://doi.org/10.1109/efta.2007.44167 83

[6]Galambos, Péter, et al. "Design, programming and orchestration of heterogeneous manufacturing systems through VR-powered remote collaboration." Robotics and ComputerIntegrated Manufacturing 33 (2015): 6877.https://doi.org/10.1016/j.rcim.2014.08.012 